\title{
SOME REMARKS ON DIVISIBLE POLYHEDRAL MV-ALGEBRAS
}

\author{
Serafina Lapenta \\ (Communicated by Anatolij Dvurečenskij)
}

\begin{abstract}
Building on similar notions for MV-algebras, polyhedral DMV-algebras are defined and investigated. For such algebras dualities with suitable categories of polyhedra are established, and the relation with finitely presented Riesz MV-algebras is investigated. Via hull-functors, finite products are interpreted in terms of hom-functors, and categories of polyhedral MV-algebras, polyhedral DMV-algebras and finitely presented Riesz MV-algebras are linked together. Moreover, the amalgamation property is proved for finitely presented DMV-algebras and Riesz MV-algebras, and for polyhedral DMV-algebras.

Mathematical Institute Slovak Academy of Science
\end{abstract}

\section{Introduction}

One of the main results in the theory of MV-algebras is the so-called Marra-Spada duality. The duality states that the full subcategory of semisimple MV-algebras is equivalent to the opposite category of closed subsets of Tychonoff cubes and definable maps. When one specialize it to finitely presented MV-algebras, one gets that the full subcategory of finitely presented MV-algebras is equivalent to the opposite of the category of rational polyhedra with $\mathbb{Z}$-maps, see Section 2 . Although the seminal idea of a deep connection between MV-algebras and polyhedra can be found in previous works by many different authors (one can see [13] and the references within), the duality is proved in [10,11].

This valuable (and fairly recent) result shed new light on MV-algebras, since it allowed a transfer of knowledge from logic to algebraic geometry and back. The duality can be naively described as a functor that maps each closed set $C \subseteq[0,1]^{\kappa}$ into the algebra obtained by restricting the functions of the free $\kappa$-generated MV-algebra to $C$. Note that in the Marra-Spada duality $\kappa$ can be an arbitrary cardinal, possibly infinite, while the algebras obtained by considering (closed) polyhedra in finite-dimensional hypercubes are called polyhedral MV-algebras and they are investigated in 1 .

Another major line of research in the framework of MV-algebras stemmed out from a simple remark: the standard example of an MV-algebra - that is the unit interval $[0,1]$ - is closed with respect to the product of real numbers. Thus, several researchers have investigated expansions of MV-algebras powerful enough to axiomatize this product. Many different notions have arisen, and we shall focus our attention on DMV-algebras and Riesz $M V$-algebras. Intuitively, they are MV-algebras endowed with a scalar multiplication, where the scalars are taken in $[0,1] \cap \mathbb{Q}$ in the former case and in $[0,1]$ in the latter.

This hierarchy of expansions of MV-algebras is analyzed from the point of view of category theory and universal algebra in 8,9], providing several pairs of adjoint functors that connect all

2010 Mathematics Subject Classification: Primary 06D35; Secondary 15A69.

Keywords: polyhedral MV-algebras, DMV-algebras, Riesz MV-algebras, tensor product, amalgamation, Łukasiewicz logic, MV-algebras.

O Open Access. (C) 2017 The Author(s), published by De Gruyter. (cc)B

This work is licensed under the Creative Commons Attribution 4.0 Internationa License. 
the most studied categories of MV-algebras with scalar product. These adjunctions, defined via tensor product of semisimple MV-algebras, can be considered hull-functors, since they provide the Riesz hull and the divisible hull of a semisimple MV-algebra, see Section 2.

Among all different results on expansions of MV-algebras, what is crucial for this note is the fact that a suitable version of the Marra-Spada duality is proved for both the categories of finitely presented DMV-algebras and finitely presented Riesz MV-algebras. Thus, the present investigation is devoted to generalize the notion of polyhedral MV-algebras to the setting of DMV-algebras and to explore the connections with finitely presented MV-algebras, DMV-algebras and Riesz MV-algebras. In Section 3 we define polyhedral DMV-algebras, we use the hull-functors to connect the various classes of polyhedral algebras, we prove dualities with appropriate classes of polyhedra, and we prove equivalences among some of the categories of algebras aforementioned. In Section 4 we define several hom-functors and we analyze, by tensor product, the interrelations of all algebras considered in the paper. We further prove the amalgamation property for finitely presented DMV-algebras and finitely presented Riesz MV-algebras, as well as for polyhedral DMV-algebras.

Through the paper we will fundamentally use the results in 1, 3, $4,8,9,9$ to make proofs as concise as possible. Therefore, we urge the interested reader to consult these references for a more detailed account of the topic.

\section{MV-algebras with scalar product and their dualities}

In what follows, MV, DMV and RMV will denote the usual algebraic categories of MV-algebras, divisible MV-algebras and Riesz MV-algebras respectively.

We briefly recall that Riesz MV-algebras are obtained by endowing MV-algebras with a scalar multiplication, modeled as a family of unary operators, with scalars taken in $[0,1]$. DMV-algebras (or divisible MV-algebras) can be obtained by taking scalars in $[0,1]_{Q}=[0,1] \cap \mathbb{Q}$. It is well known that free objects in $\mathbf{M V}, \mathbf{D M V}$ and $\mathbf{R M V}$ exist and they are algebras of $[0,1]$-valued piecewise linear functions. We shall denote them by $M V_{n}, D M V_{n}$ and $R M V_{n}$ when they are $n$-generated. We recall that the linear pieces that define $M V_{n}, D M V_{n}$ and $R M V_{n}$ are affine linear functions with integer, rational and real coefficients respectively. One can see [2,5,7] for further details.

The full subcategories of $\mathbf{M V}, \mathbf{D M V}$ and $\mathbf{R M V}$ whose objects are semisimple and finitely presented algebras have been investigated by several authors, producing dualities and adjuctions for them. To fix our notation, we shall give details on the adjuctions and dualites relevant for this note. For each category $\mathbf{C}$ used in this note, $\mathbf{C}_{\mathbf{s s}}$ will denote the full subcategory whose objects are semisimple algebras, while $\mathbf{C}_{\mathbf{f p}}$ will denote the full subcategory whose objects are finitely presented algebras.

We recall that, in our framework, an important characterization can be given for semisimple objects. Indeed, an (MV, DMV or Riesz MV)-algebra $A$ is semisimple if, and only if, it is isomorphic to a subalgebra of $C(\operatorname{Max}(A))$, continuous [0,1]-valued functions defined over the compact Hausdorff space of maximal ideals of $A$, endowed with the appropriate algebraic structure.

In 8 the authors connect the categories $\mathbf{M} \mathbf{V}_{\mathbf{s s}}$ and $\mathbf{R} \mathbf{M} \mathbf{V}_{\mathbf{s s}}$ by adjunction. The key ingredient is the semisimple tensor product of MV-algebras defined by D. Mundici in 12 . Given $A, B \in \mathbf{M V}_{\mathbf{s s}}$, their semisimple tensor product is the semisimple MV-algebra $A \otimes B$ together with a universal map $\beta_{A, B}: A \times B \rightarrow A \otimes B$ in the category $\mathbf{M V}_{\mathbf{s s}}$. Moreover, if $X$ denotes $\operatorname{Max}(A)$ and $Y$ denotes $\operatorname{Max}(B)$, up to isomorphism,

$$
A \otimes B=\langle a \cdot b \mid a \in A \subseteq C(X), \quad b \in B \subseteq C(Y)\rangle_{M V} \subseteq C(X \times Y),
$$

where, in general, for every set $X,\langle X\rangle_{M V}$ denotes the MV-algebra generated by $X$, while $a$. $b$ denotes the usual product between functions. We remark that in 12 the tensor product of 
MV-algebras has been defined in the non-semisimple case as well but, using the non-semisimple definition, it does not preserve semisimplicity. For this reason, in this note we will only consider the semisimple tensor product. Making fundamental use of Equation (1), the functor $\mathcal{T}_{\otimes}: \mathbf{M V}_{\mathbf{s s}} \rightarrow$ $\mathbf{R M V}_{\mathbf{s s}}$ has been defined in 8 on semisimple algebras by $A \mapsto[0,1] \otimes A$. It provides an adjont to the forgetful functor. The analogous result is proved in 9 for semisimple DMV-algebras, where the functor that gives the adjunction is $\mathcal{D}_{\otimes}: \mathbf{M} \mathbf{V}_{\mathbf{s s}} \rightarrow \mathbf{D M} \mathbf{V}_{\mathbf{s s}}$, defined on objects by $A \mapsto[0,1]_{Q} \otimes A$. The algebras $[0,1] \otimes A$ and $[0,1]_{Q} \otimes A$ are respectively the Riesz hull and the divisible hull of the MV-algebra $A$.

A duality between semisimple MV-algebras and closed subsets of Tychonoff cubes is obtained in [10], where the subcategory of closed spaces considered is endowed with definable maps as morphisms. Without discussing definable maps in general, we point out that in the case of finitely generated semisimple MV-algebras, the corresponding closed sets are subsets of finite-dimensional cubes, while definable maps became finite tuples of piecewise linear functions with integer coefficients. Moreover, when one considers finitely presented MV-algebras, this duality can be further specialized to rational polyhedra in finite-dimensional hypercubes [11]. We recall that an (MV, DMV or Riesz MV)-algebra $A$ is finitely presented if it is isomorphic to a quotient of a free finitelygenerated (MV, DMV or Riesz MV)-algebra by a principal ideal. Since the duality for finitely presented algebras will be used subsequently, let us describe it in more detail.

Following the notations of [13], for $0<k \leq n$, a $k$-simplex in $[0,1]^{n}$ is the convex hull $C$ of $k+1$ affinely independent points $\left\{v_{o}, \ldots, v_{k}\right\}$ in the euclidean space $[0,1]^{n}$; the points $v_{i}$ are called vertexes and $C$ is rational if every vertex has rational coordinates. A (rational) polyhedron is the union of finitely many (rational) simplexes. If $P \subseteq[0,1]^{n}$ is a polyhedron, a $\mathbb{Z}$-map $\eta: P \rightarrow[0,1]^{m}$ is a continuous map $\eta=\left(\eta_{1}, \ldots, \eta_{m}\right)$ where any $\eta_{i}:[0,1]^{n} \rightarrow[0,1]$ is a piecewise linear function with integer coefficients, that is, $\eta_{i} \in M V_{n}$ for any $i=1, \ldots, m$. If RatPol ${ }^{\mathbb{Z}}$ is the category of rational polyhedra and $\mathbb{Z}$-maps, the duality with $\mathbf{M} \mathbf{V}_{\mathbf{f p}}$ is given by the following functor:

$$
\begin{aligned}
\mathcal{M}: & \mathbf{R a t P o l}^{\mathbb{Z}} \rightarrow \mathbf{M V}_{\mathbf{f p}} \\
& \mathcal{M}(P)=M V_{n}(P)=\left\{f: P \rightarrow[0,1] \mid f \in M V_{n}\right\} \quad \text { for } P \subseteq[0,1]^{n}, \\
& \mathcal{M}(\lambda): \mathcal{M}(Q) \rightarrow \mathcal{M}(P), \quad \mathcal{M}(\lambda)(f)=f \circ \lambda .
\end{aligned}
$$

This duality is generalized to the case of DMV-algebras and Riesz MV-algebras in 9 and [6] respectively. Indeed, in [6] the full subcategory of finitely presented Riesz MV-algebras is proved to be dual to the category of finite-dimensional polyhedra and $\mathbb{R}$-maps, that is continuous maps $\lambda=\left(\lambda_{1}, \ldots, \lambda_{m}\right): P \subseteq[0,1]^{n} \rightarrow Q \subseteq[0,1]^{m}$, where $\lambda_{i} \in R M V_{n}$. Analogously, in 9$]$ the full subcategory of finitely presented DMV-algebras is proved to be dual to the category of finitedimensional rational polyhedra and $\mathbb{Q}$-maps $\lambda=\left(\lambda_{1}, \ldots, \lambda_{m}\right): P \subseteq[0,1]^{n} \rightarrow Q \subseteq[0,1]^{m}$, with $\lambda_{i} \in D M V_{n}$. Moreover, in 1$]$ the abovementioned functor is considered in the case of a generic polyhedron: the authors define the notion of polyhedral $M V$-algebras as algebras of type $M V_{n}(P)$, where $P \subseteq[0,1]^{n}$ is any polyhedron. An adaptation of the functor $\mathcal{M}$ gives a duality between polyhedral MV-algebras and polyhedra endowed with $\mathbb{Z}$-map.

\section{Polyhedral algebras}

In the sequel we generalize the definition of polyhedral MV-algebras to the case of divisible algebras. We further characterize polyhedral DMV-algebras and finitely presented Riesz MV-algebras as hulls of polyhedral MV-algebras and we prove categorical equivalences among these classes of algebras and with categories of polyhedra. 
Definition 3.1. A polyhedral DMV-algebra is a DMV-algebra such that, for some $n \in \mathbb{N}$, it is isomorphic to $D M V_{n}(P)$, where $P \subseteq[0,1]^{n}$ is a polyhedron.

Polyhedral Riesz MV-algebras could be analogously defined as Riesz MV-algebras that are isomorphic with some $R M V_{n}(P)$, where $P$ is a polyhedron in $[0,1]^{n}$. By the results in [1], these algebras are exactly the finitely presented Riesz MV-algebras.

The following lemma generalizes [4. Theorem 3.3].

Proposition 3.2. Let $P \subseteq[0,1]^{n}$ be a polyhedron. Then $[0,1]_{Q} \otimes M V_{n}(P) \simeq D M V_{n}(P)$ and $[0,1] \otimes M V_{n}(P) \simeq R M V_{n}(P)$.

Proof. In the case of Riesz MV-algebras, the claim is exactly [1: Theorem 2.3]. We give some details of the proof for DMV-algebras. It follows from [9. Theorem 3.3] that $D M V_{n} \simeq[0,1]_{Q} \otimes M V_{n}$, and from the definition of $\otimes$ we get that for any $f \in M V_{n}$, for any $\alpha \in[0,1]_{Q}$ and for any polyhedron $P \subseteq[0,1]^{n},\left.(\alpha \otimes f)\right|_{P}=\alpha\left(\left.f\right|_{P}\right)$. Since elements of type $\alpha \otimes f$ are the generators of $[0,1]_{Q} \otimes M V_{n} \simeq D M V_{n}$, we infer that $f \in D M V_{n}(P)$ if and only if $f \in[0,1]_{Q} \otimes M V_{n}(P)$.

Corollary 3.3. (i) $D$ is a polyhedral DMV-algebra if, and only if, it is the divisible hull of a polyhedral $M V$-algebra.

(ii) $R$ is a finitely presented Riesz MV-algebra if, and only if, it is the Riesz hull of a polyhedral $M V$-algebra.

Proof. It is proved in 8,9 that the algebras $[0,1]_{Q} \otimes A$ and $[0,1] \otimes A$ are respectively the divisible hull and the Riesz hull of $A$. Thus, the result is a straightforward consequence of Lemma 3.2 and the definition of polyhedral MV-algebras.

Remark. Lemma 3.2 and Corollary 3.3 entail that polyhedral DMV-algebras can be equivalently obtained by endowing polyhedral MV-algebras with divisibility operators. We note that the divisibility operators can be added by means of scalar product, as proven in 9 . Corollary 2.1 and Proposition 3.1].

In what follows, for each category of interest $\mathbf{C}, \mathbf{C}_{\mathbf{p o l y}}$ will denote the full subcategory whose objects are polyhedral algebras.

By [4: Theorem 3.3], the hull functors $\mathcal{D}_{\otimes}$ and $\mathcal{T}_{\otimes}$ map finitely presented algebras in finitely presented algebras. Moreover, $\mathcal{D}_{\otimes}: \mathbf{M V}_{\mathbf{f p}} \rightarrow \mathbf{D} \mathbf{M} \mathbf{V}_{\mathbf{f p}}$ is an essentially surjective functor, while $\mathcal{T}_{\otimes}: \mathbf{M V}_{\mathrm{fp}} \rightarrow \mathbf{R M V}_{\mathrm{fp}}$ is not. Corollary 3.3 entails that $\mathcal{D}_{\otimes}: \mathbf{M V}_{\text {poly }} \rightarrow \mathbf{D M} \mathbf{M V}_{\text {poly }}$ and $\mathcal{T}_{\otimes}: \mathbf{M V}_{\text {poly }} \rightarrow \mathbf{R} \mathbf{M} \mathbf{V}_{\mathbf{f p}}$ are both well defined and essentially surjective functors. With some restriction on arrows, we can obtain equivalences. To do so, let us fix some notations.

Let $P \subseteq[0,1]^{n}$ and $Q \subseteq[0,1]^{m}$ be polyhedra, and let $\iota_{P}^{D}: M V_{n}(P) \rightarrow D M V_{n}(P)$ and $\iota_{Q}^{R}$ : $M V_{m}(Q) \rightarrow R M V_{m}(Q)$ be the canonical embeddings of the tensor product, that can be defined via Lemma 3.2. Consider the following categories:

(i) $\mathbf{D M V} \mathbf{V}_{\text {poly }}^{\mathbf{r}}$. It is the subcategory of $\mathbf{D M V} \mathbf{V}_{\text {poly }}$ that has the same objects of $\mathbf{D} \mathbf{M} \mathbf{V}_{\text {poly }}$, while arrows are maps $f: D M V_{n}(P) \rightarrow D M V_{m}(Q)$ such that $f\left(\iota_{P}^{D}\left(M V_{n}(P)\right) \subseteq \iota \iota_{Q}^{D}\left(M V_{m}(Q)\right)\right.$.

(ii) $\mathbf{R} \mathbf{M} \mathbf{V}_{\mathbf{f p}}^{\mathbf{r}}$. It is the subcategory of $\mathbf{R} \mathbf{M} \mathbf{V}_{\mathbf{f p}}$ that has the same objects of $\mathbf{R} \mathbf{M} \mathbf{V}_{\mathbf{f p}}$, while arrows are homomorphisms $f: R M V_{n}(P) \rightarrow R M V_{m}(Q)$ such that $f\left(\iota_{P}^{R}\left(M V_{n}(P)\right) \subseteq \iota_{Q}^{R}\left(M V_{m}(Q)\right)\right.$.

Theоrem 3.4. The categories $\mathbf{M V}_{\text {poly }}$, $\mathbf{D M} \mathbf{V}_{\text {poly }}^{\mathbf{r}}$ and $\mathbf{R} \mathbf{M V}_{\mathrm{fp}}^{\mathbf{r}}$ are equivalent, with equivalences given by $\mathcal{D}_{\otimes}$ and $\mathcal{T}_{\otimes}$.

P r o of. By Corollary 3.3 the functors $\mathcal{D}_{\otimes}$ and $\mathcal{T}_{\otimes}$ are essentially surjective. Fullness follows from 4: Lemma 3.9] and the definition of arrows in $\mathbf{D} \mathbf{M} \mathbf{V}_{\mathbf{p o l y}}^{\mathbf{r}}$ and $\mathbf{R} \mathbf{M} \mathbf{V}_{\mathrm{fp}}^{\mathbf{r}}$. The proof of faithfulness is easily deduced by the fact that $A$ embeds in $\mathcal{D}_{\otimes}(A)$ and $\mathcal{T}_{\otimes}(A)$ for any $A$, see also the proof of [4: Theorem 3.5]. 
A different characterization of polyhedral MV-algebras is given in 1: Theorem 2.4]. Indeed, it is proved that an MV-algebra is polyhedral if, and only if, it is isomorphic with a finitely generated MV-subalgebra of $[0,1] \otimes M V_{n}(P)$, for some rational polyhedron $P \subseteq[0,1]^{n}$. Generalizing the characterization to our setting, we obtain the following result in a more direct manner.

Proposition 3.5. $D$ is a polyhedral DMV-algebra if, and only if, it is isomorphic to a finitely generated DMV-subalgebra of some finitely presented Riesz $M V$-algebra $R M V_{n}(P)$, with $P$ rational polyhedron.

Proof. If $D$ is a polyhedral DMV-algebra, by Corollary 3.3 and 1: Theorem 2.4] we can assume that $D \simeq \mathcal{D}_{\otimes}(A)$ and $A$ is a finitely generated subalgebra of $[0,1] \otimes M V_{n}(P)$ for some rational polyhedron $P$. It easily seen that $D \subseteq[0,1]_{Q} \otimes\left([0,1] \otimes M V_{n}(P)\right) \simeq[0,1] \otimes M V_{n}(P)$. Since $D$ is generated (as a DMV-algebra) by $\{1 \otimes a \mid a \in A\}$ and $A$ is finitely generated in $[0,1] \otimes M V_{n}(P)$, the claim is settled.

Viceversa, let $D$ be a finitely generated DMV-subalgebra of $R M V_{n}(P) \simeq[0,1] \otimes M V_{n}(P)$, where $P$ is a rational polyhedron. Since $[0,1] \otimes M V_{n}(P)$ is generated (as a Riesz MV-algebra) by $\left\{1 \otimes f \mid f \in M V_{n}(P)\right\}$, there are $\left\{f_{1}, \ldots f_{p}\right\} \subseteq M V_{n}(P)$ such that $D$ is the DMV-algebra generated by $\left\{1 \otimes f_{1}, \ldots, 1 \otimes f_{p}\right\}$. Let $A$ be the MV-algebra generated by $\left\{f_{1}, \ldots, f_{p}\right\}$. By definition and $[9$. Proposition 3.3] we infer that $D=[0,1]_{Q} \otimes A$, and that $A$ is a finitely generated MV-subalgebra of $R M V_{n}(P)$. Thus, $D$ is a polyhedral DMV-algebra by Corollary 3.3

Notation. Let $\mathbf{P o l}{ }^{\mathbb{Q}}$ and $\mathbf{P o l}{ }^{\mathbb{R}}$ denote the categories whose objects are polyhedra lying in some finite-dimensional unit cube and whose morphisms are $\mathbb{Q}$-maps and $\mathbb{R}$-maps respectively. We stress the fact that vertexes in $\mathbf{P o l}{ }^{\mathbb{Q}}$ and $\mathbf{P o}{ }^{\mathbb{R}}$ are not necessarily rationals, while we recall that $\mathbb{Q}$-maps and $\mathbb{R}$-maps are defined at the end of Section 2

Let us consider the following functors:

$$
\begin{aligned}
\mathcal{P}_{D}: & \mathbf{P o l}^{\mathbb{Q}} \rightarrow \mathbf{D M V}_{\text {poly }} \\
& \mathcal{P}_{D}(P)=D M V_{n}(P) \quad \text { for } P \subseteq[0,1]^{n}, \\
& \mathcal{P}_{D}(\eta)(f)=f \circ \eta \quad \text { for any } \mathbb{Q} \text {-map } \eta: P \rightarrow Q . \\
\mathcal{P}_{R}: & \mathbf{P o l}^{\mathbb{R}} \rightarrow \mathbf{R M V}_{\mathbf{f p}} \\
& \mathcal{P}_{R}(P)=R M V_{n}(P) \quad \text { for } P \subseteq[0,1]^{n}, \\
& \mathcal{P}_{R}(\eta)(f)=f \circ \eta \quad \text { for any } \mathbb{R} \text {-map } \eta: P \rightarrow Q .
\end{aligned}
$$

The functor $\mathcal{P}_{R}$ is exactly the one that establishes a categorical duality between the categories $\mathbf{P o l}^{\mathbb{R}}$ and $\mathbf{R} \mathbf{M} \mathbf{V}_{\mathbf{f p}}$, as proved in $[6]$. Moreover, via this duality we can easily see that indeed the objects in $\mathbf{R} \mathbf{M} \mathbf{V}_{\mathbf{f p}}$ are exactly polyhedral Riesz MV-algebras.

TheOREM 3.6. The functor $\mathcal{P}_{D}$ establishes a categorical duality between the category $\mathbf{P o l}^{\mathbb{Q}}$ of polyhedra and $\mathbb{Q}$-maps, and the category $\mathbf{D M V}_{\text {poly }}$ of polyhedral DMV-algebras.

Proof. The proof can be easily deduced from the proof of [1: Theorem 3.3]. Let us provide a sketch of it.

The functor $\mathcal{P}_{D}$ is a controvariant hom-functor and therefore well defined. Moreover, it essentially surjective by the very definition of objects in $\mathbf{D M} \mathbf{V}_{\text {poly }}$.

If $\eta_{1}, \eta_{2}: P \subseteq[0,1]^{n} \rightarrow Q \subseteq[0,1]^{m}$ are two $\mathbb{Q}$-maps such that $\eta_{1} \neq \eta_{2}$, then there exists $p \in P$ such that $\left(q_{1}, \ldots, q_{m}\right)=\eta_{1}(p) \neq \eta_{2}(p)=\left(s_{1}, \ldots, s_{m}\right)$ and therefore $q_{i} \neq s_{i}$ for some $i=1, \ldots, m$. Thus, $\mathcal{P}_{D}\left(\eta_{1}\right)\left(\pi_{i}\right)(p)=q_{i} \neq s_{i}=\mathcal{P}_{D}\left(\eta_{2}\right)\left(\pi_{i}\right)(p)$ and $\mathcal{P}_{D}\left(\eta_{1}\right) \neq \mathcal{P}_{D}\left(\eta_{2}\right)$, which entails the faithfulness of the functor. 
To prove the fullness of $\mathcal{P}_{D}$, let $h: \mathcal{P}_{D}(Q) \rightarrow \mathcal{P}_{D}(P)$ be an arrow. For $i=1, \ldots, m$, take $\eta_{i}=h\left(\left.\pi_{i}\right|_{Q}\right) \in D M V_{n}(P)$ and consider the map $\eta=\left(\eta_{1}, \ldots, \eta_{m}\right): P \rightarrow[0,1]^{m}$. Since $\left\{\pi_{1}, \ldots \pi_{m}\right\}$ is a set of generators for $D M V_{m}$, for any $\alpha \in D M V_{m}, h\left(\left.\alpha\right|_{Q}\right)=h\left(\tau\left(\left.\pi_{1}\right|_{Q},\left.\ldots \pi_{m}\right|_{Q}\right)\right)$, where $\tau$ is an MV-term. Therefore, since $h$ is a homomorphism, $h\left(\left.\alpha\right|_{Q}\right)=\tau\left(\eta_{1}, \ldots, \eta_{m}\right)=\left.(\alpha \circ \eta)\right|_{P}$. We now prove that $\eta(p) \in Q$ for any $p \in P$. By [1: Lemma 3.2], if $x \notin Q$ there exists a $\mathbb{Z}$ map $\lambda:[0,1]^{m} \rightarrow[0,1]$ (which is also a $\mathbb{Q}$-map) such that $\lambda(Q)=0$ and $\lambda(x) \neq 0$. Therefore $h\left(\left.\lambda\right|_{Q}\right)=0=\left.(\lambda \circ \eta)\right|_{P}$. Thus $x \notin \eta(P)$, since $\lambda(x) \neq 0$ while $\lambda(\eta(P))=0$. Finally, it is easily seen that $\mathcal{P}_{D}(\eta)=h$.

The analogous of Proposition 3.5 is used in 1 as a stepping stone to provide a duality between compact sets in finite-dimensional unit cubes and finitely generated and semisimple MV-algebras, which was also proved in the case of arbitrary (i.e. non necessarely finitely generated) semisimple MV-algebras in [10. The same result holds for Riesz MV-algebras and it is proved in 6: Theorem 3.1]. With analogous arguments and some technicalities proved in [9. Section 4], the following holds.

TheOREM 3.7. The functor $\mathcal{P}_{D}$ can be generalized to a duality between the category $\mathbf{K}_{\mathbb{Q}}$ of closed sets in some finite-dimensional unit cube and $\mathbb{Q}$-maps and the full subcategory $\mathbf{D} \mathbf{M} \mathbf{V}_{\text {fgs }}$ of finitely generated and semisimple DMV-algebras.

Proof. We provide a sketch of the proof. Consider the functor $\mathcal{C}: \mathbf{K}_{\mathbb{Q}} \rightarrow \mathbf{D M V}_{\text {fgs }}$ defined by $\mathcal{C}(C)=D M V_{n}(C)$ and $\mathcal{C}(\eta)(f)=f \circ \eta$, for any $C \subseteq[0,1]^{n}, D \subseteq[0,1]^{m}$ closed sets and for any $\mathbb{Q}$-map $\eta: C \rightarrow D$. Fullness and faithfulness of the functor can be argued exactly as in Theorem 3.6, while 9. Proposition 4.1, Lemma 4.3 and Proposition 4.3] entail that the functor is essentially surjective. Indeed, any finitely generated and semisimple DMV-algebra $D \simeq D M V_{n} / I$ is isomorphic with $D M V(C)$, where $C$ is the closed set defined by $C=\left\{x \in[0,1]^{n} \mid f(x)=\right.$ 0 for any $f \in I\}$.

\section{Hull-functors, Hom-functors and polyhedral algebras}

Theorem 3.6 shows that a given category of polyhedral algebras is dual to an appropriate category of polyhedra, by a functor that essentially is a hom-functor. We shall now analyze the relations between hom-functors in categories of polyhedra and hull-functors.

Notation. Let $Q$ be a fixed polyhedron. In the sequel we denote by $\mathbf{H}_{\mathbb{Z}}(-, Q): \mathbf{P o l}^{\mathbb{Z}} \rightarrow$ Set the standard hom-functor in $\mathbf{P o l}^{\mathbb{Z}}$, by $\mathbf{H}_{\mathbb{Q}}(-, Q): \mathbf{P o l}{ }^{\mathbb{Q}} \rightarrow$ Set the hom-functor in $\mathbf{P o l}{ }^{\mathbb{Q}}$ and by $\mathbf{H}_{\mathbb{R}}(-, Q): \mathbf{P o l}{ }^{\mathbb{R}} \rightarrow$ Set the hom-functor in $\mathbf{P o l}^{\mathbb{R}}$. Moreover, for any $n \in \mathbb{N}, C_{n}$ will denote the unit cube $[0,1]^{n}$, which is also a semisimple MV-algebra.

Notice that, with these notations, $\mathcal{P}_{D}(P)=\mathbf{H}_{\mathbb{Q}}\left(P, C_{1}\right)$ and $\mathcal{P}_{R}(P)=\mathbf{H}_{\mathbb{R}}\left(P, C_{1}\right)$.

We now characterize the above defined hom-functors in terms of finite products.

Lemma 4.1. Let $n, m \geq 1$ be natural numbers and let $P \subseteq[0,1]^{m}$ be a polyhedron. Then,

$$
\begin{aligned}
& \mathbf{H}_{\mathbb{Z}}\left(P, C_{n}\right)=\prod_{i=1}^{n} M V_{m}(P), \\
& \mathbf{H}_{\mathbb{Q}}\left(P, C_{n}\right)=\prod_{i=1}^{n} D M V_{m}(P), \\
& \mathbf{H}_{\mathbb{R}}\left(P, C_{n}\right)=\prod_{i=1}^{n} R M V_{m}(P) .
\end{aligned}
$$


P r o o f. We give the proof in the first case, the other cases being similar. Note that $\lambda \in \mathbf{H}_{\mathbb{Z}}\left(P, C_{n}\right)$ if, and only if, $\lambda=\left(\left.\lambda_{1}\right|_{P}, \ldots,\left.\lambda_{n}\right|_{P}\right)$ where $\lambda_{i} \in M V_{m}$ for any $1 \leq i \leq n$. That is, if, and only if, $\lambda \in \prod_{i=1}^{n} M V_{m}(P)$. Thus, $\mathbf{H}_{\mathbb{Z}}\left(P, C_{n}\right)=\prod_{i=1}^{n} M V_{m}(P)$ and it is semisimple since it is a product of semisimple MV-algebras.

LemMA 4.2. For any polyhedron $P \subseteq[0,1]^{m}, \mathbf{H}_{\mathbb{Z}}\left(P, C_{n}\right)$ is a polyhedral $M V$-algebra, $\mathbf{H}_{\mathbb{Q}}\left(P, C_{n}\right)$ is a polyhedral DMV-algebra and $\mathbf{H}_{\mathbb{R}}\left(P, C_{n}\right)$ is a finitely presented Riesz $M V$-algebra. Moreover, if $P$ is rational, $\mathbf{H}_{\mathbb{Z}}\left(P, C_{n}\right)$ is a finitely presented $M V$-algebra and $\mathbf{H}_{\mathbb{Q}}\left(P, C_{n}\right)$ is a finitely presented DMV-algebra.

Proof. The proof is a straightforward application of Lemma 4.1 and the duality given in Theorem 3.6. Indeed, finite products of polyhedral algebras (or finitely presented algebras) are send to finite disjoint unions of polyhedra (or rational polyhedra), which are again polyhedra (or rational polyhedra).

Building on Lemma 4.1 and Lemma 4.2 , we define the following functors:

$$
\begin{aligned}
& \mathbf{H}_{\mathbb{Z}}\left(-, C_{n}\right): \mathbf{P o l}^{\mathbb{Z}} \rightarrow \mathbf{M V}_{\text {poly }}, \\
& \mathbf{H}_{\mathbb{Q}}\left(-, C_{n}\right): \mathbf{P o l}^{\mathbb{Q}} \rightarrow \mathbf{D M V}_{\text {poly }}, \\
& \mathbf{H}_{\mathbb{R}}\left(-, C_{n}\right): \mathbf{P o l}^{\mathbb{R}} \rightarrow \mathbf{R M V}_{\text {fp }} .
\end{aligned}
$$

It is easily seen that these functors are well defined, given that they are restrictions (on both domain and codomain) of the usual controvariant hom-functors. We also notice that for $n=1$ we recover the functors that give the dualities already described for finitely presented or polyhedral MV-algebras, DMV-algebras and Riesz MV-algebras. Moreover, both $\mathbf{H}_{\mathbb{Z}}\left(-, C_{n}\right)$ and $\mathbf{H}_{\mathbb{Q}}\left(-, C_{n}\right)$ can be restricted (on both domain and codomain) to the full subcategories having rational polyhedra and finitely presented algebras as objects.

Lemma 4.3. Let $n, m$ be a natural numbers and let $P_{1}, \ldots, P_{n}$ be polyhedra in $[0,1]^{m}$. Then,

$$
\begin{aligned}
{[0,1] } & \otimes \prod_{i=1}^{n} M V_{m}\left(P_{i}\right) \simeq \prod_{i=1}^{n}\left([0,1] \otimes M V_{m}\left(P_{i}\right)\right) ; \\
{[0,1]_{Q} } & \otimes \prod_{i=1}^{n} M V_{m}\left(P_{i}\right) \simeq \prod_{i=1}^{n}\left([0,1]_{Q} \otimes M V_{m}\left(P_{i}\right)\right) .
\end{aligned}
$$

P r o of. We remark again that, via the duality already established, finite products of polyhedral algebras correspond to finite disjoint unions of polyhedra. Whence, denoted by $P$ the disjoint union of $P_{1}, \ldots, P_{n}$, the following holds:

$$
\begin{aligned}
{[0,1]_{Q} \otimes \prod_{i=1}^{n} M V_{m}\left(P_{i}\right) } & \simeq[0,1]_{Q} \otimes M V_{m}(P) \simeq D M V_{m}(P) \\
& \simeq \prod_{i=1}^{n} D M V_{m}\left(P_{i}\right) \simeq \prod_{i=1}^{n}\left([0,1]_{Q} \otimes M V_{m}\left(P_{i}\right)\right) .
\end{aligned}
$$

The case of Riesz MV-algebras is analogous.

Proposition 4.4. With the above notations, for any $n, m \geq 1$ and for any polyhedron $P \subseteq[0,1]^{m}$,

$$
\mathbf{H}_{\mathbb{Q}}\left(P, C_{n}\right) \simeq \mathcal{D}_{\otimes}\left(\mathbf{H}_{\mathbb{Z}}\left(P, C_{n}\right)\right) \quad \text { and } \quad \mathbf{H}_{\mathbb{R}}\left(P, C_{n}\right) \simeq \mathcal{T}_{\otimes}\left(\mathbf{H}_{\mathbb{Z}}\left(P, C_{n}\right)\right) .
$$


Pr o of. Let $P$ be a fixed polyhedron in $[0,1]^{m}$. For $n=1$, by Lemma 3.2 and Corollary 3.3 ,

$$
\begin{aligned}
& \mathbf{H}_{\mathbb{Q}}\left(P, C_{1}\right)=D M V_{m}(P) \simeq \mathcal{D}_{\otimes}\left(M V_{m}(P)\right)=\mathcal{D}_{\otimes}\left(\mathbf{H}_{\mathbb{Z}}\left(P, C_{1}\right)\right) \\
& \mathbf{H}_{\mathbb{R}}\left(P, C_{1}\right)=R M V_{m}(P) \simeq \mathcal{T}_{\otimes}\left(M V_{m}(P)\right)=\mathcal{T}_{\otimes}\left(\mathbf{H}_{\mathbb{Z}}\left(P, C_{1}\right)\right)
\end{aligned}
$$

For $n>1$, the same argument applies via Lemma 4.1 and Lemma 4.3. In the case of $\mathcal{D}_{\otimes}$ :

$$
\begin{aligned}
\mathbf{H}_{\mathbb{Q}}\left(P, C_{n}\right) & =\prod_{i=1}^{n} D M V_{m}(P) \simeq \prod_{i=1}^{n}[0,1]_{Q} \otimes M V_{m}(P) \\
& \simeq[0,1]_{Q} \otimes \prod_{i=1}^{n} M V_{m}(P)=\mathcal{D}_{\otimes}\left(\mathbf{H}_{\mathbb{Z}}\left(P, C_{n}\right)\right) .
\end{aligned}
$$

We now prove the analogous of Proposition 4.4 on arrows. The proof is a straightforward consequence of the universal property of the hull-constructions, but it requires a clear display of all the algebras involved in the picture.

Proposition 4.5. Let $f: P \subseteq[0,1]^{m} \rightarrow Q \subseteq[0,1]^{k}$ be an arrow in $\mathbf{P o l}^{\mathbb{Z}}$. With the previous notations, for any $n \geq 1$,

$$
\mathbf{H}_{\mathbb{Q}}\left(f, C_{n}\right)=\mathcal{D}_{\otimes}\left(\mathbf{H}_{\mathbb{Z}}\left(f, C_{n}\right)\right) \quad \text { and } \quad \mathbf{H}_{\mathbb{R}}\left(f, C_{n}\right)=\mathcal{T}_{\otimes}\left(\mathbf{H}_{\mathbb{Z}}\left(f, C_{n}\right)\right) .
$$

P r o of. Let us prove the claim for $\mathcal{D}_{\otimes}$, the other case being similar. To ease our notation, we shall identify each polyhedron and each arrow in $\mathbf{P o l}^{\mathbb{Z}}$ with its copy in $\mathbf{P o l}{ }^{\mathbb{Q}}$. Moreover, we shall denote by $f^{\mathbb{Z}}$ the map $\mathbf{H}_{\mathbb{Z}}\left(f, C_{n}\right)$, by $f^{\mathbb{Q}}$ the map $\mathbf{H}_{\mathbb{Q}}\left(f, C_{n}\right)$, by $\iota_{P}$ the embedding of $\mathbf{H}_{\mathbb{Z}}\left(P, C_{n}\right)$ in $\mathbf{H}_{\mathbb{Q}}\left(P, C_{n}\right)$ and by $\iota_{Q}$ the embedding of $\mathbf{H}_{\mathbb{Z}}\left(Q, C_{n}\right)$ in $\mathbf{H}_{\mathbb{Q}}\left(Q, C_{n}\right)$, that exist by Proposition 4.4 and the various definitions of hulls. In order to prove our claim, we need to show that $f^{\mathbb{Q}}$ satisfies the universal property that uniquely characterizes $\mathcal{D}_{\otimes}\left(f^{\mathbb{Z}}\right)$, that is

$$
\iota_{P} \circ f^{\mathbb{Z}}=\mathcal{D}_{\otimes}\left(f^{\mathbb{Z}}\right) \circ \iota_{Q}
$$

Thus, for any $\lambda \in \mathbf{H}_{\mathbb{Z}}\left(Q, C_{n}\right)$,

$$
\text { and } \quad \begin{aligned}
\iota_{P}\left(f^{\mathbb{Z}}(\lambda)\right) & =\iota_{P}(\lambda \circ f)=\mathbf{1} \cdot(\lambda \circ f)=\lambda \circ f \\
& f^{\mathbb{Q}}\left(\iota_{Q}(\lambda)\right)=f^{\mathbb{Q}}(\mathbf{1} \cdot \lambda)=f^{\mathbb{Q}}(\lambda)=\lambda \circ f .
\end{aligned}
$$

Therefore $\iota_{P} \circ f^{\mathbb{Z}}=f^{\mathbb{Q}} \circ \iota_{Q}$ and $f^{\mathbb{Q}}=\mathcal{D}_{\otimes}\left(f^{\mathbb{Z}}\right)$. Notice that we have used twice the fact that the embedding $B \rightarrow A \otimes B$ is defined by $b \mapsto 1_{A} \otimes b$, that $a \otimes b=a \cdot b$ and that the function $\mathbf{1}$, that is identically equal to 1 , is the unit in our spaces.

The commutative diagrams in Figure 1 and Figure 2, with $\hookrightarrow$ being the inclusion functors, illustrate the relations found between the categories of polyhedra and algebras via hom-functors and tensor functors. We recall that finitely presented Riesz MV-algebras are exactly polyhedral Riesz MV-algebras, via the duality proven in [6].

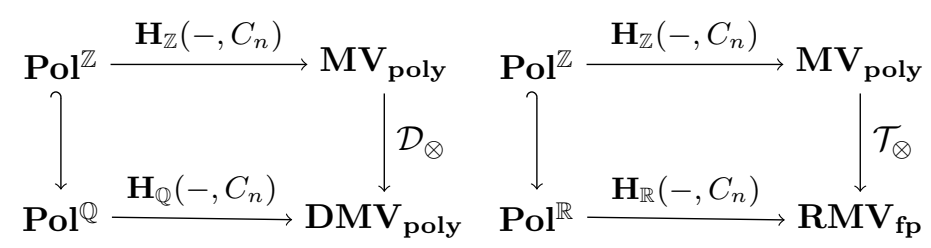

Figure 1. Tensor product and polyhedral algebras 


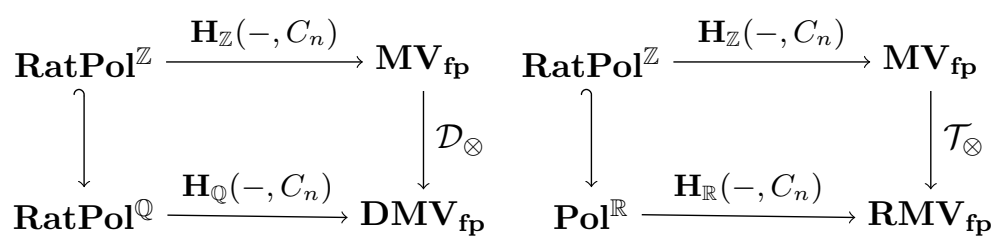

FIGURE 2. Tensor product and finitely presented algebras

We conclude this note applying the results obtained in Section 3 to prove the amalgamation property for polyhedral DMV-algebras, finitely presented DMV-algebras and finitely presented Riesz MV-algebras in a quite straightforward manner.

Proposition 4.6. The subcategories $\mathbf{D M V} \mathbf{V}_{\mathbf{f p}}, \mathbf{D M V}_{\text {poly }}$ and $\mathbf{R} \mathbf{M} \mathbf{V}_{\mathbf{f p}}$ of $D M V$-algebras and Riesz MV-algebras, whose objects are polyhedral or finitely presented algebras, have the amalgamation property.

P r o of. The proof is a consequence of Lemma 3.2. We give all details in one case, the others being similar.

Let $A, B, Z$ be polyhedral DMV-algebras such that $Z$ embeds in both $A$ and $B$, with embeddings $z_{A}$ and $z_{B}$. Consider the MV-algebraic reduct of $A$ and $B$. By the amalgamation property of polyhedral MV-algebras, 1: Theorem 4.2], there exist a polyhedral MV-algebra $M$ and two embeddings of MV-algebras $f_{A}, f_{B}$ such that $f_{A}: A \hookrightarrow M, f_{B}: B \hookrightarrow M$. By [1: Theorem 3.3] there exists a polyhedron $P \subseteq[0,1]^{n}$ such that $M \simeq M V_{n}(P)$ and by Lemma 3.2 $[0,1]_{Q} \otimes M V_{n}(P) \simeq$ $D M V_{n}(P)$. Moreover, the algebra $M V_{n}(P)$ embeds in $[0,1]_{Q} \otimes M V_{n}(P)$. Thus, we have the diagram represented in Figure 3 .

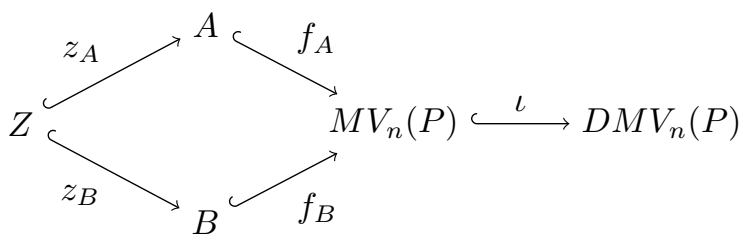

Figure 3. Amalgamation property

By the amalgamation property of polyhedral MV-algebras, $f_{A} \circ z_{A}=f_{B} \circ z_{B}$, therefore the diagram commutes. Since all algebras are semisimple, all composite morphisms are indeed homomorphisms of DMV-algebras, see 9. Lemma 3.1].

The other cases follow similarly using the amalgamation property of finitely presented MV-algebras, 13: Corollary 6.7], Lemma 3.2. [4. Theorem 3.3] and the fact that any homomorphism of MV-algebras between semisimple Riesz MV-algebras is a homomorphism of Riesz MV-algebras, see [14. Proposition 11.53].

Conclusions and acknowledgments. This work builds on [1,4,10, with the aim of analyzing the interactions between MV-algebras, DMV-algebras and Riesz MV-algebras in the context of duality with polyhedra, using tensor product as key tool. The results show a different way to build polyhedral or finitely presented algebras by hom-functors and tensors, and they are a step forward in the program of understanding more deeply the interrelations of these categories of MV-algebras. 
The author is grateful to the referee for many helpful suggestions that improved the clarity of the work, and to Ioana Leuşean, for all the interesting discussions on the subject of this note.

\section{REFERENCES}

[1] BUSAniChe, M.-CABRER, L. M.-MUNDiCI, D.: Polyhedral MV-algebras, Fuzzy Sets and Systems 292 (2016), 150-159.

[2] Cignoli, R.-D'OtTaViano, I. M. L.-MUNDICI, D.: Algebraic foundation of many valued reasoning, Trends Log. 7, Springer Netherlands, 2000.

[3] DIACONESCU, D.-LEUŞTEAN, I.: The Riesz Hull of a semisimple MV-algebra, Math. Slovaca (special issue in honor of Antonio Di Nola) 65(4) (2015), 801-816.

[4] DI NOLA, A.-LAPENTA, S.-LEUŞTEAN, I.: An analysis of the logic of Riesz spaces with strong unit, Ann. Pure Appl. Logic 169(3) (2018), 216-234.

[5] DI NOLA, A.-LEUŞTEAN, I.: Lukasiewicz logic and Riesz Spaces, Soft Comput. 18(12) (2014), $2349-2363$.

[6] DI NOLA, A.-LENZI, G.-VITALE, G.: Riesz-McNaughton functions and Riesz MV-algebras of nonlinear functions, Fuzzy Sets and Systems 311 (2017), 1-14.

[7] GERLA, B.: Rational Eukasiewicz logic and Divisible MV-algebras, Neural Networks World 10(11) (2001), $579-584$.

[8] LAPENTA, S.-LEUŞTEAN, I.: Scalar extensions for the algebraic structures of Lukasiewicz logic, J. Pure Appl. Algebra 220 (2016), 1538-1553.

[9] LAPENTA, S.-LEUŞTEAN, I.: Notes on divisible MV-algebras, Soft Comput. 21(21) (2017), 6213-6223.

[10] MARRA, V.-SPADA, L.: The Dual Adjunction between $M V$-algebras and Tychonoff Spaces, Studia Logica 100(1-2) (2012), 253-278.

[11] MARRA, V.-SPADA, L.: Duality, projectivity and unification in Eukasiewicz logic and MV-algebras, Ann. Pure Appl. Logic 164(3) (2013), 192-210.

[12] MUNDICI, D.: Tensor products and the Loomis-Sikorski theorem for MV-algebras, Adv. Pure Appl. Math. 22 (1999), 227-248.

[13] MUNDICI, D.: Advanced Lukasiewicz calculus and MV-algebras. Trends Log. 35, Springer, 2011.

[14] SCHECHTER, E.: Handbook of Analysis and Its Foundations, Academic Press, Inc., San Diego, CA, 1997.

Received 1. 2. 2019

Accepted 5. 7. 2019
Department of Mathematics University of Salerno

Via Giovanni Paolo II, 132

84084 Fisciano (SA)

ITALY

E-mail: slapenta@unisa.it 